\title{
Measurement of single top production in the tW channel and search for $s$ channel in pp collisions
}

\author{
Mario Merola* for the CMS Collaboration \\ INFN, Napoli (IT) \\ E-mail: mario.meroladcern.ch
}

\begin{abstract}
Measurements of single top quark production in the $\mathrm{tW}$ and s channels in pp collisions are presented, based on data collected in 2012 at a centre-of-mass energy of $8 \mathrm{TeV}$. In the tW channel a top quark is produced in association with a $\mathrm{W}$ boson. The experimental signature is similar to top pair production, and there is interference at higher orders between the two processes. The measurement is perfomed using final states in which the associated $\mathrm{W}$ boson as well as the one originating from the top quark decay leptonically. Multivariate methods are used to extract the cross section and the result is compared with current standard model theory predictions. Multivariate techniques are also adopted in the search for $s$-channel single top quark production in order to discriminate the very small signal from the huge contribution of background processes. In both analyses the signal contribution is determined together with the background estimation by performing a maximum-likelihood fit to the Boosted Decision Trees discriminants in signal and background enriched phase space regions.
\end{abstract}

The European Physical Society Conference on High Energy Physics 22-29 July 2015

Vienna, Austria

${ }^{*}$ Speaker. 

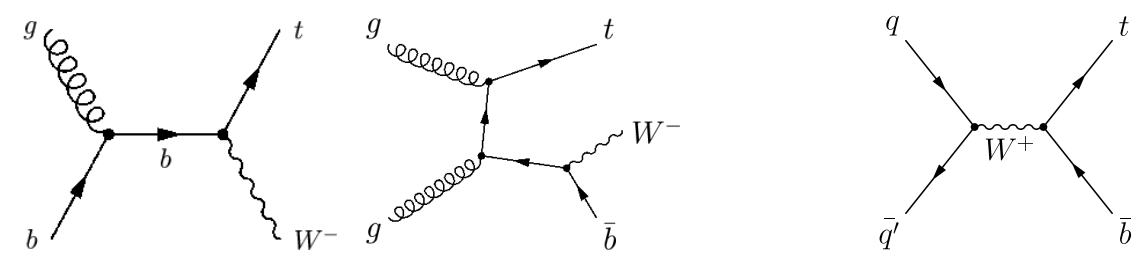

Figure 1: Representative leading order (LO) Feynman diagrams in the five- and four-flavour schemes for single top production in the tW channel (left) and in the $s$ channel (right).

\section{Introduction}

The top quark, discovered at the Tevatron proton anti-proton collider in 1995 [1, 2], can be produced via strong interaction in association with the top anti-quark and via electroweak interaction as single top. Three are the different single top production modes, the $t$ channel, the $s$ channel and the tW production. In Figure 1 the Feynman diagrams for the tW and $s$ channel processes are shown. In addition to testing the SM prediction at the electroweak scale, the single top production allows to probe directly the $W t b$ vertex, potentially detecting non-SM couplings ( $t$ and $\mathrm{tW}$ channels) and is sensitive to models of new physics involving non-SM mediators ( $s$ channel).

In this contribution the first observation of the tW associated production and the search for the $s$-channel single top production are presented.

\section{Single top production in association with $\mathrm{W}$ boson}

The first observation within CMS [3] of tW production analysing data corresponding to an integrated luminosity of $12.2 \mathrm{fb}^{-1}$ collected at a centre-of-mass energy of $8 \mathrm{TeV}$ is described [4]. The theoretical prediction for the production cross section of this process is $\sigma_{t W-\text { ch. }}^{\text {th }}=22.2 \pm$ 0.6 (scale) \pm 1.4 (PDF) pb [5]. Since at the NLO the definition of tW production mixes with top quark pair production (well illustrated by the second diagram in Figure 1), the 'diagram removal' scheme [6], in which all doubly resonant NLO tW diagrams are removed, has been adopted to define the signal.

The analysis considers the dilepton decay channels, so the final state is composed of two oppositely charged muons or electrons, a $\mathrm{b}$ jet and two neutrinos. The primary background comes from $\mathrm{t} \overline{\mathrm{t}}$ production and $\mathrm{Z} / \gamma^{\star}$ events. Electrons and muons are required to have $p_{\mathrm{T}}>20 \mathrm{GeV} / \mathrm{c}$ and $|\eta|<2.5$ (electrons), $<2.4$ (muons) and the dilepton invariant mass, $m_{l l}$, is required to be greater than $20 \mathrm{GeV} / c^{2}$. In addition, for same flavour leptons events are rejected if $81<m_{l l}<101 \mathrm{GeV} / c^{2}$ and $H_{\mathrm{T}}<50 \mathrm{GeV}$. Selected jets, reconstructed by clustering particle flow candidates [7] using the anti- $k_{T}$ algorithm [8], must be within $|\eta|<2.4$ and have $p_{\mathrm{T}}>30 \mathrm{GeV} / c$. Jets coming from hadronization of $b$ quarks are tagged based on the Combined Secondary Vertex algorithm [9]. The signal region is identified requiring exactly 1 jet being b tagged $(1 j 1 t)$, while two control regions enriched in tit background are defined as well, as $2 j 1 t$ and $2 j 2 t$.

The analysis makes use of a multivariate technique, based on boosted decision trees (BDT) [10], in order to distinguish the $\mathrm{tW}$ signal from the dominant $\mathrm{t} \overline{\mathrm{t}}$ background. The BDT analyzer is trained using 13 variables among which the ones related to the loose jets (jets with loose $p_{\mathrm{T}}$ and $|\eta|$ re- 

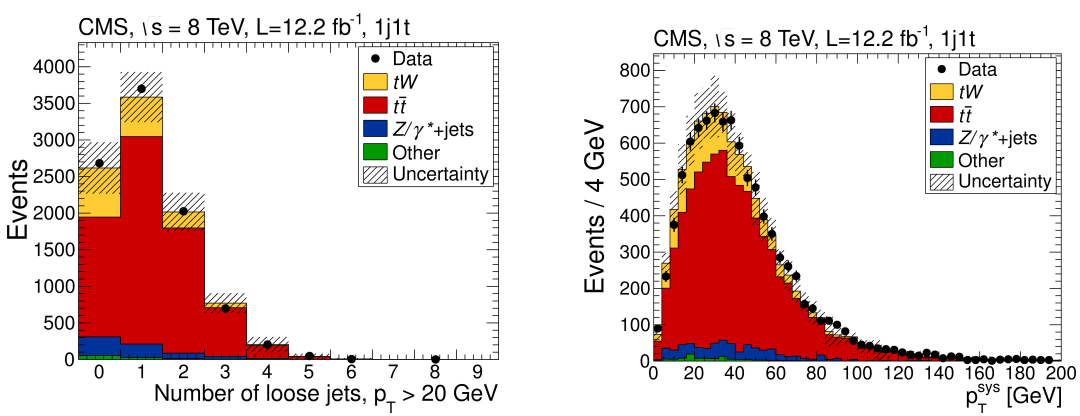

Figure 2: Number of loose jets in the event (left) and magnitude of the vector sum of the transverse momenta of leptons, jets and $\mathscr{E}_{\mathrm{T}}$ (right) in the signal region.
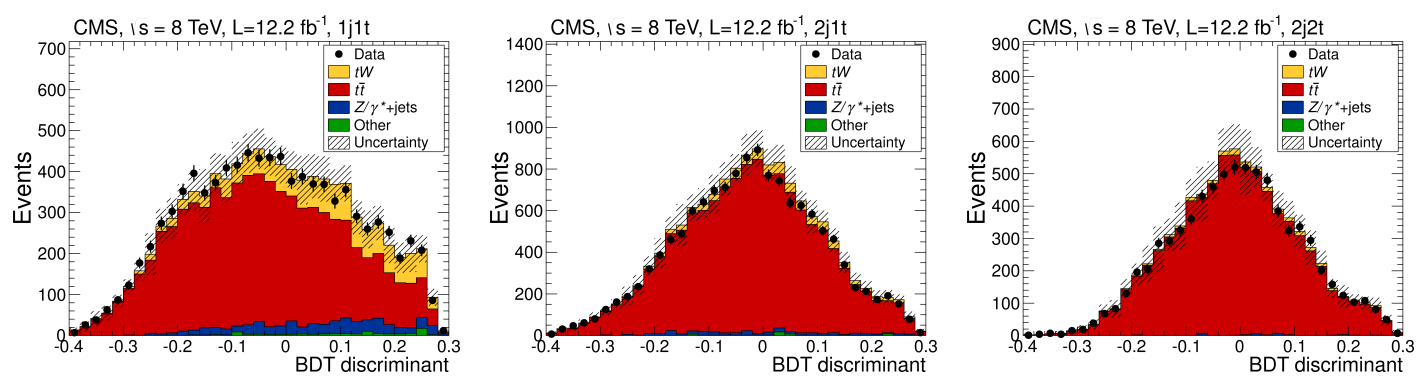

Figure 3: BDT discriminant in $1 j 1 t$ (left), $2 j 1 t$ (centre) and $2 j 2 t$ (right) event categories.

quirements) and to the sum of transverse momenta of leptons, jets and missing energy in the event have the highest discrimination power (see Figure 2).

The signal extraction is performed with a simultaneous binned likelihood fit of the BDT discriminant in the three regions, where the signal and backgrounds distributions are taken from simulation and their rates are allowed to vary in the fit, constrained in the likelihood function by the systematic uncertainties (affecting both normalisation and shape of the BDT discriminant).

The analysis measures a cross section of $23.4 \pm 5.4 \mathrm{pb}$, where the main contributions to the uncertainty come from the choice of the thresholds for the matrix element and parton showering matching in simulation of têt production (14\%) and the renormalisation and factorisation scales $(12 \%)$. The significance of the events is found to be $6.1 \sigma$ away from the background-only hypothesis corresponding to a $p$ value of $5 \times 10^{-10}$.

In Figure 3 the BDT discriminant distributions in the three phase space regions normalised to the fit results are shown.

From the cross section a measurement of the CKM element $\left|V_{\mathrm{tb}}\right|$ can be obtained. Assuming $\left|V_{\mathrm{td}}\right|$ and $\left|V_{\mathrm{ts}}\right|<<\left|V_{\mathrm{tb}}\right|$ we have $\left|V_{\mathrm{tb}}\right|=\sqrt{\sigma_{t W-\mathrm{ch} .} / \sigma_{t W-\mathrm{ch} .}^{\mathrm{th}}}$ and measure:

$$
\left|V_{\mathrm{tb}}\right|=1.03 \pm 0.12 \text { (exp.) } \pm 0.04 \text { (th.) }
$$

where $\sigma_{t W-\text { ch. }}^{\text {th }}$ is the SM prediction calculated assuming $\left|V_{\mathrm{tb}}\right|=1$.

Furthermore, to test the robustness and stability of the present result, two cross-check analyses are performed as well. The first one involves a single variable fit rather than the BDT discriminant 
while the second is based only on event counts after selection. Both analyses measure a cross section in agreement with the BDT analysis, but with larger uncertainties.

\section{Search for single top production in the $s$ channel}

The single top production in the $s$ channel at the LHC is unfavoured with respect to the other two production modes, involving a quark and an anti-quark in the initial state. The SM prediction at NNLL order for this process at the LHC and at $8 \mathrm{TeV}$ is $\sigma_{s-\mathrm{ch}}^{\text {th }}=5.55 \pm 0.08$ (scale) \pm $0.21(\mathrm{PDF}) \mathrm{pb}$ [5]. Nonetheless the interest in this channel is focused on possible enhancement of its production due to new physics involving non-SM mediators, like $\mathrm{W}^{\prime}$ or charged Higgs boson [11]. The study presented here [12] analyses $19.3 \mathrm{fb}^{-1}$ of data collected by CMS in 2012 at a centre-of-mass energy $\sqrt{s}=8 \mathrm{TeV}$.

The final state topology is characterized by the presence of one muon or electron and two $b$ quarks, one coming from the top-quark decay and one recoiling against the top quark. The event selection considers exactly one isolated muon or one electron with $p_{\mathrm{T}}>26 \mathrm{GeV} / c,|\eta|<2.1$ and $p_{\mathrm{T}}>30 \mathrm{GeV} / c,|\eta|<2.5$, respectively. Exactly two jets are required to have $p_{\mathrm{T}}>40 \mathrm{GeV} / c$ for $|\eta|<4.5$ and events with additional jets with $p_{\mathrm{T}}>30 \mathrm{GeV} / c$ are rejected. The two selected jets are also required to be originated from $b$ quarks, adopting the track counting algorithm with high purity requirement. Due to the ambiguity in the reconstruction of the top quark hypothesis from $b$ jet, lepton and $\mathscr{H}_{\mathrm{T}}$, two top mass hypotheses are defined and the correct b jet-to-top assignment is taken based on the one which gives the closer invariant top mass to the value $172.5 \mathrm{GeV} / \mathrm{c}^{2}$.

At the end the signal sample is still dominated by the backgrounds, mainly $\mathrm{tt}, \mathrm{W}+\mathrm{jets}$ and $t$ channel single top, and so a multivariate analysis using BDT is performed in order to take advantage of the different final state topology of the signal and the backgrounds. Two independent BDT trainings are set up in the signal region $(2 j 2 t)$ and in a $t \bar{t}$ enriched region $(3 j 2 t)$, optimised for electron and muon decay channels. Among the kinematic and angular variables considered, the ones with the highest discriminating power are the vector sum of $p_{\mathrm{T}}$ of the two b-jets system and the $\mathrm{W}$ boson transverse mass (Figure 4). For the first variable the signal shows a lower $p_{\mathrm{T}}$ distribution with respect to $t \bar{t}$ since the two $b$ jets carry a larger fraction of the original transverse momentum, peaking around zero. The $\mathrm{W}$ transverse mass, reconstructed from the lepton and the $\mathbb{H}_{\mathrm{T}}$, shows a clear jacobian peak for lepton-neutrino pairs coming from the same W-boson decay (signal), while presents a broader distribution for events where two $\mathrm{W}$ bosons are produced (tit).

The signal extraction consists in a binned maximum likelihood fit to the BDT distributions on data in the $2 j 2 t$ and $3 j 2 t$ categories simultaneously (Figure 5 ). The inclusion of the $3 j 2 t$ category allows to constrain the $\mathrm{t} \overline{\mathrm{t}}$ and $\mathrm{W}+$ jets backgrounds while taking into account all possible correlations in the systematics uncertainties between the two regions. The signal and all the background models are taken from simulation except for the multijet QCD background, for which a dedicated treatment has been adopted. The QCD model is taken from a control region in data defined inverting the lepton isolation for muons and requiring that either the isolation cut or identification criteria fail for electrons. The QCD normalisation is estimated by a fit to the $m_{\mathrm{T}}$ (muons) or $\boldsymbol{H}_{\mathrm{T}}$ (electrons) and its uncertainty is conservatively assigned by repeating the same fit in a QCD-enriched region and varying the non-QCD contributions by $20 \%$. 

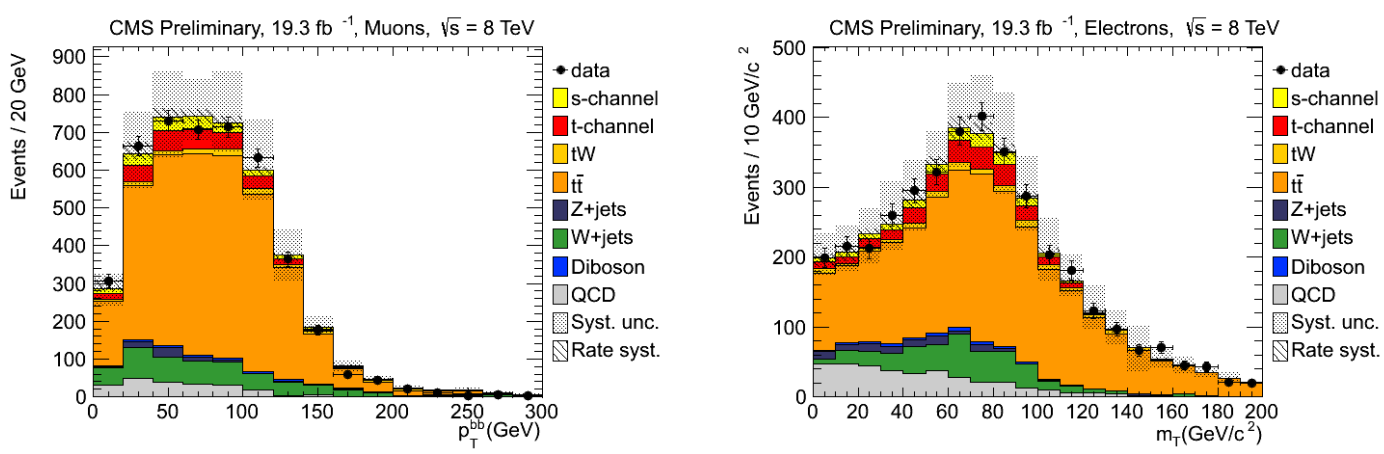

Figure 4: Two of the most discriminating variables used in the BDT for separating signal and backgrounds, in the signal enriched event category: vector sum of $p_{\mathrm{T}}$ of the two b-jets system (left) and the $\mathrm{W}$ boson transverse mass (right).
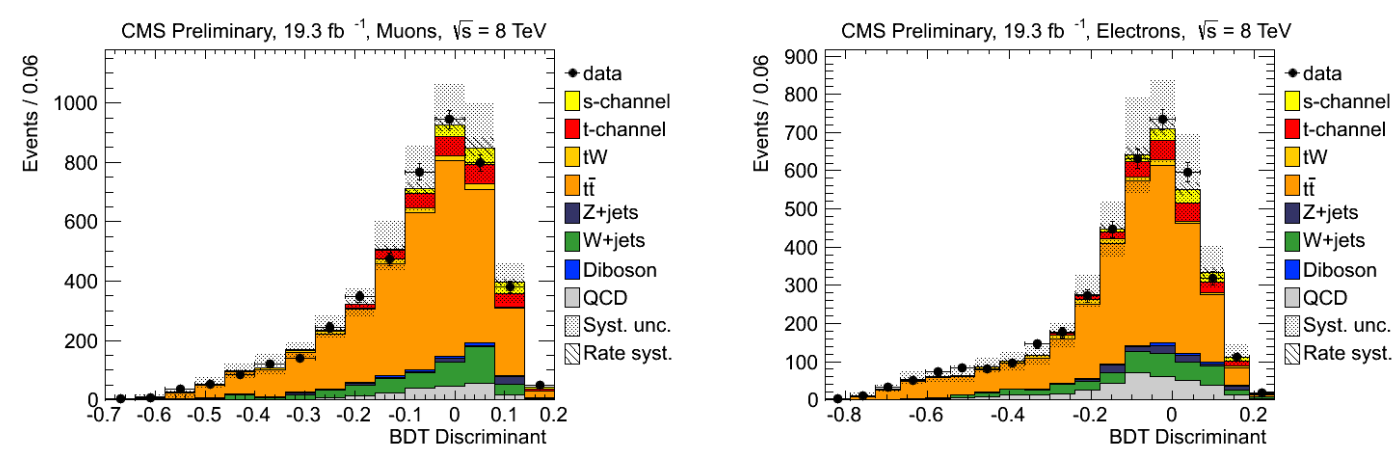

Figure 5: BDT discriminant for the muon (left) and electron (right) channels in the signal enriched event category. The simulation is normalised to the fit results.

The likelihood model includes the background rates as nuisance parameters with a coefficient for each background template with a log-normal prior. The prior uncertainties on the background normalisation have been taken from the recent measurements of the corresponding processes, i.e. $10 \%$ for $\mathrm{t} \overline{\mathrm{t}}, 30 \%$ for $\mathrm{W}+\mathrm{jets}, 10-30 \%$ for QCD (in the different event categories), 15\% for single top in the $t$ channel. The impact of the background rates uncertainties on the $s$-channel cross section measurement is evaluated removing one uncertainty at a time from the likelihood model and measuring the corresponding variation in the profile likelihood. The other instrumental and theoretical uncertainties (as jet energy scale and resolution, b-tagging efficiencies, matching thresholds, renormalisation and factorisation scales, PDFs, etc.) are evaluated performing two fits to the pseudodatasets generated with the up/down variations of the systematic source of interest (with other sources fixed to zero), and taking the difference in the fitted signal yield between these fits and the nominal fit as the corresponding up/down uncertainties.

The systematic uncertainties with the highest impact on the measurement have been found to be the factorisation and renormalisation scales $(\sim 80 \%)$, jet energy scales $(\sim 50 \%)$, and matching thresholds $(\sim 30 \%)$. The single top-quark $s$-channel inclusive production cross section has been 


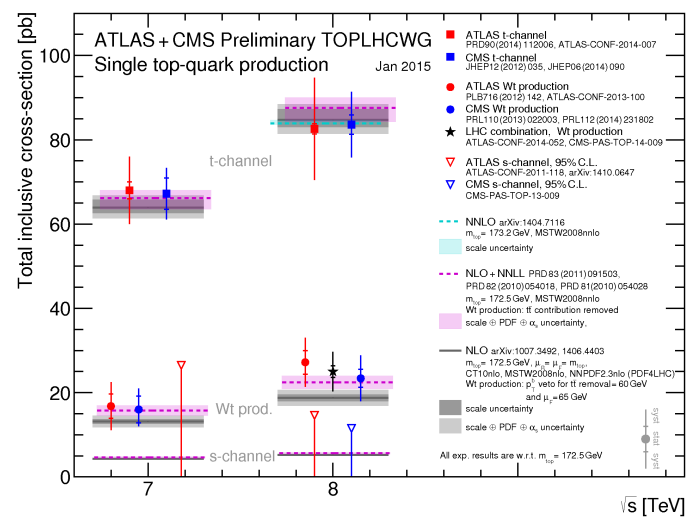

Figure 6: Summary of single top cross section measurements performed by CMS and ATLAS compared with theoretical prediction.

measured to be $\sigma_{s-\text { ch. }}=6.2 \pm 5.4$ (exp.) \pm 5.9 (th.) pb. Using the Feldman-Cousins unified approach [13] the $68 \%$ confidence level (CL) intervals which do not reach negative cross section values are reported as well: $\sigma_{s-\mathrm{ch} .}=6.2_{-5.1}^{+8.0} \mathrm{pb}$. Finally, the measurement leads to an upper limit evaluated using a Bayesian approach of $11.5(17.0,9.0) \mathrm{pb}$ at $95 \% \mathrm{CL}$, where the numbers in parenthesis refer to the expected limits in presence of SM signal and in the background-only hypothesis.

Future extensions of this analysis will include the whole $7+8 \mathrm{TeV}$ datasets to increase the sensitivity of the measurement, will expoit other background enriched phase space regions and the reduced factorisation/renormalisation scale uncertainties from the next-to-leading order $t \bar{t}$ generators.

\section{Conclusions}

In this paper the results of the most recent studies on single top production at CMS in the tW and $s$ channels have been described. The first analysis, the tW inclusive cross section measurement, with its signal significance of $6.1 \sigma$ represents the first observation of this production mechanism at the LHC. The measurement is also used to extract a $\left|V_{\mathrm{tb}}\right|$ CKM matrix element determination. The second analysis constitutes the first search for the single top production in the $s$ channel at CMS, measuring an upper limit on the cross section of $11.5 \mathrm{pb}$ at $95 \% \mathrm{CL}$.

All the results in single top sector are summarised in Figure 6 where the cross section measurements are compared with the theoretical predictions.

\section{References}

[1] CDF Collaboration, Observation of Top Quark Production in $\bar{p} p$ Collisions with the Collider Detector at Fermilab, Phys. Rev. Lett. 74 (1995) 2626

[2] D0 Collaboration, Observation of the Top Quark, Phys. Rev. Lett. 74 (1995) 2632

[3] CMS Collaboration, The CMS experiment at the CERN LHC, JINST 03 (2008) S08004

[4] CMS Collaboration, Observation of the Associated Production of a Single Top Quark and a W Boson in pp Collisions at $\sqrt{s}=8 \mathrm{TeV}$, Phys. Rev. Lett. 112 (2014) 231802 
[5] Kidonakis, N., NNLL threshold resummation for top-pair and single-top production, Phys. Part. Nucl. 45 (2014) 714 [hep-ph/1210. 7813]

[6] Frixione, S. and Laenen, E. and Motylinski, P. and White, C. and Webber, B. R., Single-top hadroproduction in association with a W boson, JHEP 07 (2008) 029 [hep-ph / 0805 . 3067 ]

[7] CMS Collaboration, Particle-Flow Event Reconstruction in CMS and Performance for Jets, Taus, and $\not_{\mathrm{T}}$, CMS Physics Analysis Summary CMS-PAS-PFT-09-001 (2009)

[8] Cacciari, M. and Salam, G. P. and Soyez, G., The anti-k t jet clustering algorithm, JHEP 04 (2008) 063

[9] CMS Collaboration, Identification of b-quark jets with the CMS experiment, JINST 8 (2013) P04013

[10] Breiman, L. and Friedman, J. and Stone, C. J. and Olshen, R. A., Classification and Regression Trees, Chapman and Hall, 1984.

[11] Tait, T. M. P. and Yuan, C. P., Single top quark production as a window to physics beyond the standard model, Phys. Rev. D 63 (2000) 014018

[12] CMS Collaboration, Search for s-channel single top-quark production in pp collisions at $\sqrt{s}=8 \mathrm{TeV}$, CMS Physics Analysis Summary CMS-PAS-TOP-13-009 (2013)

[13] Feldman, G. J. and Cousins, R. D., Unified approach to the classical statistical analysis of small signals, Phys. Rev. D 57 (1998) 3873 